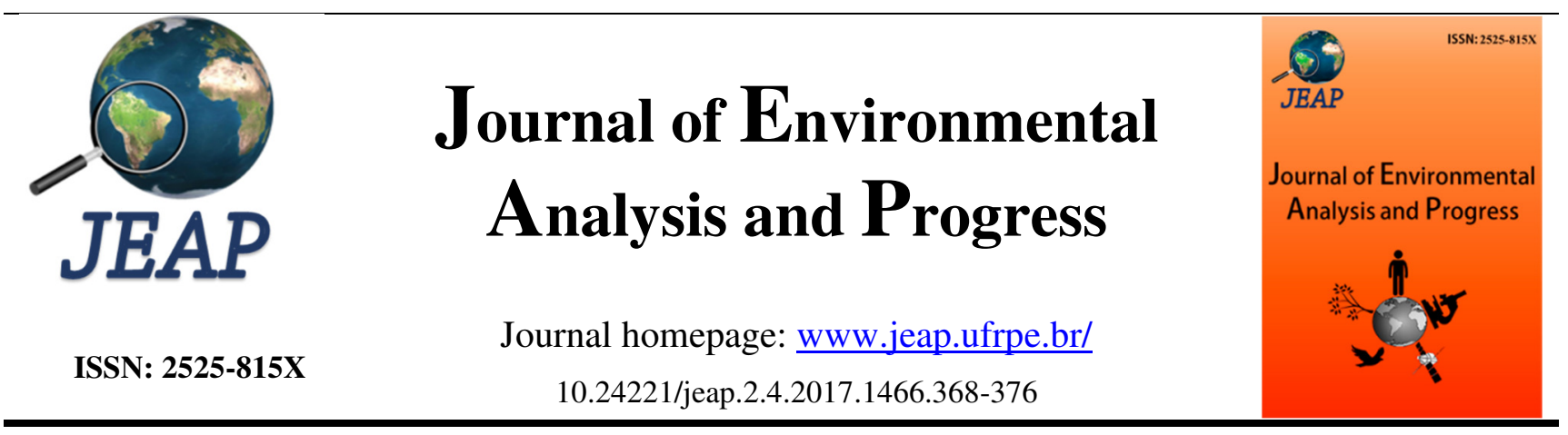

\title{
Variabilidade espacial da sazonalidade da chuva no semiárido brasileiro
}

\section{Spatial variability of rainfall seasonality in semi-arid region of Brazil}

\author{
Wagner Martins dos Santos ${ }^{\mathrm{a}}$, Rodolfo Marcondes Silva Souza ${ }^{\mathrm{b}}$, Eduardo Soares de Souza ${ }^{\mathrm{a}}$, André
} Quintão de Almeida ${ }^{\mathrm{c}}$, Antonio Celso Dantas Antonino ${ }^{\mathrm{b}}$

a Universidade Federal Rural de Pernambuco-UFRPE, Unidade Acadêmica de Serra Talhada-UAST. Av. Gregório Ferraz Nogueira, S/N, Serra Talhada, Pernambuco, Brasil. CEP: 56909-535. E-mail: wagnnermds@gmail.com, eduardo.souza.rd@gmail.com.

b Observatório Nacional da Dinâmica da Água e de Carbono no Bioma Caatinga, Universidade Federal de PernambucoUFPE, Departamento de Energia Nuclear-DEN, Av. Prof. Luiz Freire, 1000, Cidade Universitária, Recife-PE. CEP: 50740-545. E-mail: rodolfo.marcondes@ufpe.br, acdantonino@gmail.com.

c Universidade Federal de Sergipe-UFS, Departamento de Engenharia Agrícola-DEAGRI, Cidade Universitária, Avenida Marechal Rondon, S/N, Jardim Rosa Elze, São Cristóvão-SE. CEP: 49100-000. E-mail: andreqa@gmail.com.

\section{A R T I C L E I N F O}

Recebido 30 Jun 2017 Aceito 04 Ago 2017

Publicado 15 Ago 2017

\begin{abstract}
A B S T R A C T
The rainfall is one of the key regulators of biogeochemical processes of a region, and the understanding of its spatial variability and seasonality throughout the year is fundamental to the management of water resources, and agricultural planning of the Brazilian semi-arid region. Seasonal variability was evaluated from the rain in the Brazilian semi-arid region under three conditions: i) the monthly average of the time series, ii) wettest year (2009) and the driest year (2012). Daily rainfall data was obtained from the database of the National Water Agency (ANA) and were calculated monthly and annual total rainfall, the relative entropy $\left(D_{k}\right)$, the index of seasonality $\left(S_{k}\right)$, the characteristic time of rain $\left(C_{k}\right)$ and the duration of the rainy season $\left(Z_{k}\right)$. These variables were spaced to semi-arid region so to enable the interpretation of spatial variability from geostatistics techniques. The magnitude of the coefficient of variation of the variables was similar to the average of the time series, the wettest year and driest year, except for the $C_{k}$ and the $Z_{k}$ in the driest year. The spherical model of semivariogram was the one of the best represented the spatial variability of most of the variables studied. In the central part of the semiarid, even in the rainiest year, there were the smallest annual values of rainfall. The statistical measures of seasonality and interannual variability of the rainfall used in this study showed a satisfactory performance in the characterization of the behavior of rains in the semi-arid region of Brazil.
\end{abstract}

Keywords: Relative entropy, geostatistic, kriging.

\section{R E S U M O}

A chuva é um dos principais reguladores dos processos biogeoquímicos de uma região e o entendimento da sua variabilidade espacial e da sua sazonalidade ao longo ano, é fundamental para o gerenciamento dos recursos hídricos e planejamento agrícola do Semiárido brasileiro. Foi avaliada a variabilidade sazonal da chuva no Semiárido brasileiro sob três condições: i) a média mensal das séries temporais, ii) ano considerado mais chuvoso (2009) e o ano considerado mais seco (2012). Informações diárias de chuva foram obtidos da base de dados da Agência Nacional de Águas (ANA) e a partir deles, foram calculados os valores totais precipitados nos meses e no ano, a entropia relativa $\left(D_{k}\right)$, o índice de sazonalidade 
$\left(S_{k}\right)$, o tempo característico da chuva $\left(C_{k}\right)$ e a duração da estação chuvosa $\left(Z_{k}\right)$. Essas variáveis foram espacializadas para toda a região semiárida de modo à possibilitar a interpretação da variabilidade espacial a partir de técnicas de geoestatística. A magnitude do coeficiente de variação das variáveis foi semelhante entre a média das séries temporais, o ano mais chuvoso e o ano mais seco, exceto para o $C_{k}$ e a $Z_{k}$ no ano mais seco. $O$ modelo de semivariograma esférico foi quem melhor representou a variabilidade espacial da maioria das variáveis estudadas. $\mathrm{Na}$ parte central do semiárido, mesmo no ano mais chuvoso, ocorreram os menores valores anuais de chuva. As medidas estatísticas de sazonalidade e variabilidade interanual da chuva utilizadas neste estudo mostraram um desempenho satisfatório na caracterização do comportamento das chuvas na região semiárida do Brasil.

Palavras-Chave: Entropia relativa, geoestatística, krigagem, hidroclimatologia.

\section{Introdução \\ O Nordeste brasileiro ocupa 1.600 .000} $\mathrm{km}^{2}$ do território nacional, com $62 \%$ da sua área correspondente ao Polígono das Secas, uma região semiárida de $940.000 \mathrm{~km}^{2}$, que abrange nove Estados da região e enfrenta um problema crônico de falta de água e precipitação abaixo de 800 mm. ano $^{-1}$ (Marengo, 2010). A disponibilidade natural de água na região Semiárido do Brasil é caracterizada por uma distribuição de chuvas bastante irregular, baixos índices de pluviosidade e longos períodos de estiagem (Nys \& Engle, 2014; Hastenrath, 2012). Os longos períodos de estiagem, influenciados pelo El Niño (Hastenrath, 2012; Cavalcanti, 2012), ocasionam danos irreparáveis à população da região, em especial àqueles que retiram seu sustento da atividade agrícola, em especial a agricultura familiar (Rodriguez et al., 2015). Os diferentes sistemas de circulação atmosférica que atuam nessa região tornam a climatologia complexa, refletindo em uma grande variabilidade climática, considerando as chuvas, com eventos de precipitação variando no tempo e no espaço (Silva, 2004).

Assim, é importante utilizar técnicas para caracterização climática de determinadas regiões, como o uso da teoria da entropia e a utilização de métricas sazonais. Na região semiárida do Brasil, Rodriguez et al. (2015), delimitaram regiões homogêneas com base na teoria da entropia. Para caracterização dos padrões climáticos de uma região, atualmente podemos utilizar métricas sazonais que geram índices complexos como a duração da estação chuvosa, e que com sua distribuição possibilita o mapeamento da região dando uma visão como um todo de suas devidas características Feng et al. (2013). Embora o número de trabalhos com estas técnicas esteja aumentando em outros países, no Brasil, estas técnicas ainda são poucos utilizadas, principalmente em regiões de clima semiárido.

Diante do exposto acima, o objetivo deste estudo foi utilizar métricas sazonais para avaliar a variabilidade sazonal da chuva no Semiárido brasileiro tomando como base três condições: i) a média mensal das séries temporais, ii) ano considerado mais chuvoso e o ano considerado mais seco.

\section{Material e Métodos}

Localização da área de estudo

A região semiárida do Brasil está situada entre os paralelos de $3,08^{\circ}$ e $17,66^{\circ}$ de latitude sul e entre os meridianos de $35,25^{\circ}$ e $46,12^{\circ}$ de longitude a oeste do meridiano de Greenwich. Essa região está inserida, em quase sua totalidade, no Nordeste brasileiro, abrangendo os estados de Alagoas, Bahia, Ceará, Paraíba, Pernambuco, Piauí, Rio Grande do Norte e Sergipe, além do norte do Estado de Minas Gerais na região Sudeste.

No semiárido brasileiro ocorrem pelo menos três tipos climáticos da classificação de Köppen, sendo Aw, BWh e BSh. O clima semiárido da região é caracterizado por apresentar altas médias térmicas, baixa nebulosidade, elevadas taxas de evaporação e, sobretudo, baixos índices pluviométricos, em torno de 500 a 700 $\mathrm{mm}$ anuais (Oliveira et al., 2006).

\section{Aquisição dos dados}

Os dados diários de chuva (mm) foram obtidos da Agência Nacional de Águas (ANA) para toda a região semiárida. Foram selecionadas estações pluviométricas que apresentavam no mínimo 15 anos de dados em toda a região semiárida e de alguns pontos limítrofes. Os dados da zona limite do semiárido foram mantidos para diminuir os erros por interpolação e melhorar a interpretação dos mapas. Na base de dados da ANA foram encontradas 210 estações pluviométricas com registros de pelo menos 15 anos de chuva. Entre as 210 estações, o maior período de registro de dados foi de 106 anos.

A partir dos valores diários de chuva, obtiveram-se as médias mensais para cada estação. Os dados médios mensais foram utilizados para realizar a caracterização das condições de chuva média em toda a região semiárida em estudo. Em seguida, para representar o ano chuvoso e seco, foram escolhidos anos com valores totais de chuvas 
médias anuais acima e abaixo do valor médio de precipitação anual na região. Tomando como base a média $(\mu)$ e o desvio padrão $(\sigma)$ da chuva na região, classificou-se o ano como chuvoso aquele com chuva média mais próxima ou acima do intervalo $\mu+\sigma$ e o ano seco como aquele com chuva média mais próxima ou abaixo do intervalo $\mu-\sigma$.

\section{Métricas da sazonalidade}

A sazonalidade da chuva na escala mensal foi avaliada de acordo com as medidas estatísticas de sazonalidade e variabilidade interanual propostas por Feng et al. (2013). Inicialmente foi calculada a climatologia mensal da chuva pela média mensal para cada estação. Em seguida, o ano hidrológico, $\mathrm{k}$, foi definido como iniciado no primeiro mês, $\mathrm{m}$, após a menor média mensal de chuva.

O total de chuva para cada ano hidrológico, $\mathrm{R}_{\mathrm{k}}$, e a sua distribuição de probabilidade mensal, $\mathrm{p}_{\mathrm{k}, \mathrm{m}}$, são calculados como:

$$
\begin{gathered}
R_{k}=\sum_{m=1}^{12} r_{k, m} \\
p_{k, m}=\frac{r_{k, m}}{R_{k}}
\end{gathered}
$$

onde rk,m é a chuva mensal.

A entropia relativa Dk para cada ano hidrológico, foi calculada como:

$$
D_{k}=\sum_{m=1}^{12} p_{k, m} \cdot \log _{2}\left(\frac{p_{k, m}}{q_{m}}\right)
$$

onde qm a distribuição uniforme e tem valor de $1 / \mathrm{m}$ para cada mês. O índice de sazonalidade, Sk foi calculado com Dk e Rk, e normalizado pelo valor máximo do total de chuva do ano hidrológico, Rmax, considerando toda a base de dados por:

$$
S_{k}=D_{k} \cdot\left(\frac{R_{k}}{R_{\max }}\right)
$$

Para obtenção dos demais indicadores da variabilidade interanual, foram calculados: a magnitude da chuva anual $R_{k}$, o centróide da distribuição da chuva, $\mathrm{C}_{\mathrm{k}}$, para o tempo característico da estação chuvosa e a amplitude, $Z_{k}$, para a duração da estação chuvosa (mês), sendo $C_{k}$ e $Z_{k}$ utilizados como o primeiro e segundo momento da distribuição da chuva mensal:

$$
\begin{aligned}
& C_{k}=\frac{1}{R_{k}} \cdot \sum_{m=1}^{12} m \cdot r_{k, m} \\
& Z_{k}=\sqrt{\frac{1}{R_{k}} \cdot\left|m-C_{k}\right|^{2} \cdot r_{k, m}}
\end{aligned}
$$

\section{Análise de geoestatística}

A dependência espacial dos parâmetros estimados da chuva na região semiárida foi analisada por meio da geoestatística, utilizando-se o ajuste de semivariogramas. A função semivariância foi calculada para todas as direções (semivariograma isotrópico), com o objetivo de perceber a presença de dependência espacial dos dados. Previamente foram ajustados alguns modelos de semivariogramas teórico como esférico, o exponencial e o gaussiano.

A escolha do melhor ajuste foi baseada no coeficiente de determinação $\left(R^{2}\right)$ entre os valores observados e estimados pela krigagem pata cada modelo de semivariograma. Foi escolhido o modelo que apresentou maior $\mathrm{R}^{2}$. Os ajustes dos semivariogramas foram realizados pelo método do quadrado mínimo ponderado com o software $\mathrm{R}$ 3.4.0 ( $R$ Development Core Team, 2017) e o pacote geoR (Ribeiro Junior \& Diggle, 2001; Ribeiro Júnior \& Diggle, 2015).

$\mathrm{O}$ grau de dependência espacial (GDE) foi calculado a partir da proporção em porcentagem entre o efeito pepita $\left(\mathrm{C}_{0}\right)$ e o patamar, dado pela soma do efeito pepita e a variação estruturada (C). A classificação do GDE foi realizada de acordo com Cambardella et al. (1994) como: alto grau de dependência espacial (GDE < 25\%); médio grau de dependência espacial $(25 \leq \mathrm{GDE} \leq 75 \%)$ e fraco grau de dependência espacial (GDE $>75 \%$ ).

\section{Resultados}

$\mathrm{Na}$ Tabela 1 são apresentadas as estatísticas descritivas dos parâmetros estimados com base nos valores históricos de chuva e para os anos considerados seco (2012) e chuvoso (2009) na região semiárida do Brasil. A chuva média do ano 2009 foi a que mais se aproximou do limite superior do intervalo $\mu+\sigma$, representando um ano chuvoso na região semiárida do Brasil (Tabela 1). A amplitude de variação, entre os valores mínimo e máximo (Vmax - Vmin), aumentou em quase $61 \%$, no ano chuvoso, e em torno de $43 \%$, no ano seco. O valor médio de chuva no ano seco $(435 \mathrm{~mm})$ se aproximou 
bastante do valor mínimo da série histórica (398 $\mathrm{mm})$.

Ao comparar a média histórica de chuva com os anos seco e chuvoso, houve um aumento de $17 \%$ na chuva média em 2009 e uma redução de $67 \%$ na chuva média em 2012 (Tabela 1).

Tabela 1. Estatística descritiva dos valores normais climatológicos, do ano considerado chuvoso e seco, da entropia relativa $\left(D_{k}\right)$, índice de sazonalidade $\left(S_{k}\right)$, tempo característico da chuva $\left(C_{k}\right)$ e duração da estação chuvosa $\left(Z_{k}\right)$ no semiárido brasileiro.

\begin{tabular}{|c|c|c|c|c|c|c|}
\hline & & $\begin{array}{c}\text { Chuva } \\
(\mathbf{m m})\end{array}$ & $D_{k}$ & $S_{k(}$ & $C_{k}$ & $\begin{array}{c}Z_{k} \\
\text { (meses) }\end{array}$ \\
\hline \multirow{6}{*}{ Média histórica } & Média anual & 727 & 0,522 & 0,211 & 5,79 & 2,18 \\
\hline & Vmax & 1148 & 0,939 & 0,479 & 7,07 & 3,19 \\
\hline & Vmin & 398 & 0,047 & 0,020 & 4,33 & 1,54 \\
\hline & Desvio padrão & 176 & 0,249 & 0,116 & 0,56 & 0,43 \\
\hline & $\mathrm{CV}(\%)$ & 24,26 & 46,02 & 55,16 & 9,77 & 19,85 \\
\hline & $\mathrm{N}^{\circ}$ estações & $158(210)^{*}$ & - & - & - & - \\
\hline \multirow{6}{*}{ Ano chuvoso (2009) } & Média anual & 873 & 0,843 & 0,263 & 6,35 & 2,31 \\
\hline & Vmax & 2132 & 1,867 & 0,897 & 8,87 & 3,93 \\
\hline & Vmin & 203 & 0,283 & 0,053 & 2,55 & 0,97 \\
\hline & Desvio padrão & 323 & 0,261 & 0,129 & 1,01 & 0,57 \\
\hline & $\mathrm{CV}(\%)$ & 37,05 & 31,01 & 48,94 & 15,95 & 25,00 \\
\hline & $\mathrm{N}^{\circ}$ estações & $270(328) *$ & - & - & & - \\
\hline \multirow{6}{*}{ Ano seco (2012) } & Média anual & 435 & 1,388 & 0,338 & 6,98 & 2,13 \\
\hline & Vmax & 1364 & 2,934 & 0,986 & 9,51 & 4,51 \\
\hline & $\mathrm{Vmin}$ & 29 & 0,190 & 0,032 & 3,05 & 0,55 \\
\hline & Desvio padrão & 213 & 0,455 & 0,192 & 1,30 & 0,77 \\
\hline & $\mathrm{CV}(\%)$ & 49,01 & 32,82 & 57,00 & 18,63 & 36,19 \\
\hline & $\mathrm{N}^{\circ}$ estações & $251(304)^{*}$ & - & - & - & - \\
\hline
\end{tabular}

Ao analisar o tempo da estação chuvosa $\left(Z_{k}\right)$, em meses, não foi observado uma diferença entre os anos analisados. Mesmo no ano considerado chuvoso (2009), este tempo (2,31 meses) foi semelhante (2,13 meses) ao ano considerado seco (2012) e a média histórica da região (2,18 meses).

O modelo de semivariograma que apresentou o melhor resultado $\left(\mathrm{R}^{2}\right)$ para modelar a distribuição espacial da maior parte das variáveis estudadas foi o esférico (Tabela 2). $\mathrm{O}$ alcance da chuva média foi menor do que o alcance dos anos chuvoso e seco.

Os mapas de chuva anual com base na série histórica e com base nos anos seco (2012) e chuvoso (2009) podem ser visualizados na Figura 1. A chuva média anual na região semiárida, interpolada a partir da krigagem, está nas classes entre 410 e $1185 \mathrm{~mm}$ (Figura 1A).

No ano considerado como chuvoso, 2009 (Figura 1B), observa-se que na maior parte da região semiárida ocorreu uma chuva maior do que a média anual, porém observou-se, também, que, em algumas áreas, principalmente no Estado da Bahia, ocorreu uma chuva abaixo da média anual para esta área.

Analisando a Figura 1, percebe-se que em cerca de $45,4 \%$ da região semiárida ocorre, em média, chuvas entre $720-875 \mathrm{~mm}$. Tanto no ano com chuva média (Figura 1A) como no ano chuvoso (Figura 1B), a menor classe de chuva desses anos (410-565 mm) ocorre em cerca de $8,0 \%$ na parte central da região semiárida. No ano chuvoso (Figura 1B) há uma distribuição proporcional entre as classes de 565-710, 710-875 e 875-1030 mm, onde cada uma ocorre uma média de $20 \%$ da região semiárida, respectivamente.

No ano seco (Figura 1C), aproximadamente $9,0 \%$ da região semiárida teve chuva abaixo de $255 \mathrm{~mm}$ e em $34,6 \%$ da região a chuva variou entre a classe de $255-410 \mathrm{~mm}$. Essas duas classes, que não aparecem no ano com chuva média e no ano chuvoso, somam $43,6 \%$ da área semiárida.

Os mapas da distribuição espacial da entropia relativa da chuva $\left(D_{k}\right)$, índice de sazonalidade $\left(S_{k}\right)$ e duração da estação chuvosa $\left(\mathrm{Z}_{\mathrm{k}}\right)$ no Semiárido brasileiro, para chuva média da região, ano chuvoso e ano seco podem ser visualizados na Figura 2. Na série histórica de dados (Figura 2A), a maior classe de valor de entropia foi a de 0,8 a 1,0. Considerando os anos extremos (Figura 2BC), foram observadas as maiores classe de $D_{k}$, com valores acima de 0,6 , podendo chegar a 2,0 no ano considerado seco. 
Tal resultado comprova a elevada variabilidade espacial das chuvas na região Nordeste do Brasil. O mesmo comportamento pode ser observado para a variável $Z_{k}$, como a mesma distribuição espacial da $D_{k}$. Um fato interessante foram os baixos valores de duração da estação chuvosa, podendo chegar até 3,5 meses do ano, na região que se estende de norte a sul, localizada na porção leste do Semiárido.

Tabela 2. Parâmetros da análise de geoestatística para a chuva, a entropia relativa (Dk), o índice de sazonalidade (Sk), o tempo característica da chuva (Ck) e a duração da estação chuvosa (Zk).

\begin{tabular}{cccccccc}
\hline \multirow{4}{*}{ Média histórica } & Modelo & $\mathbf{C}_{\mathbf{0}}$ & $\mathbf{C}$ & $\mathbf{A}(\mathbf{k m})$ & $\mathbf{I D E}$ & $\mathbf{R}^{\mathbf{2}}$ \\
\cline { 2 - 7 } & Chuva & Exp & 28670 & 52739 & 249,6 & 35,21 & 0,5917 \\
& $D_{k}$ & Esf & 0,0 & 0,094 & 777,4 & 0,0 & 0,9423 \\
& $S_{k}$ & Esf & 0,0 & 0,022 & 790,5 & 0,0 & 0,9231 \\
& $C_{k}$ & Esf & 0,19 & 0,07 & 301,2 & 73,08 & 0,2035 \\
& $Z_{k}$ & Gau & 0,019 & 0,334 & 3955,4 & 5,45 & 0,9017 \\
& Chuva & Esf & 54308 & 117667 & 727,2 & 31,59 & 0,4776 \\
& $D_{k}$ & Esf & 0,030 & 0,054 & 543,5 & 35,71 & 0,4327 \\
& $S_{k}$ & Esf & 0,005 & 0,009 & 570,0 & 35,71 & 0,5923 \\
& $C_{k}$ & Esf & 0,760 & 0,320 & 721,2 & 70,37 & 0,2052 \\
& $Z_{k}$ & Esf & 0,090 & 0,300 & 821,9 & 23,08 & 0,6534 \\
& Chuva & Exp & 23959 & 81394 & 611,5 & 22,74 & 0,5401 \\
& $D_{k}$ & Esf & 0,08 & 0,18 & 482,4 & 30,77 & 0,5672 \\
& $S_{k}$ & Esf & 0,10 & 0,045 & 1315,9 & 18,18 & 0,6376 \\
& $C_{k}$ & Esf & 1,12 & 0,560 & 437,1 & 66,67 & 0,1820 \\
& $Z_{k}$ & Esf & 0,22 & 0,49 & 708,3 & 30,99 & 0,5694 \\
\hline
\end{tabular}

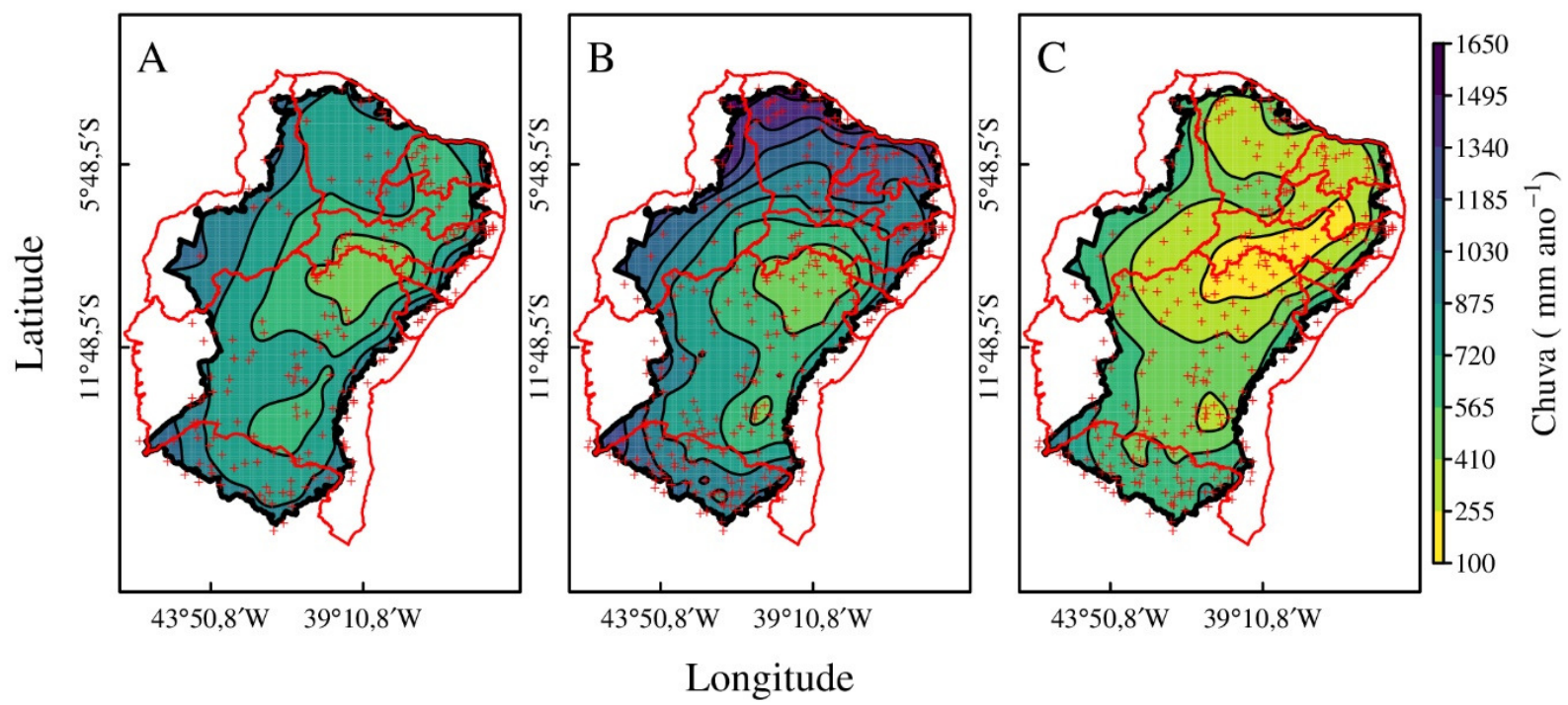

Figura 1. Distribuição espacial da chuva no Semiárido brasileiro: A: média (estações com pelo menos 15 anos de dados), B: 2009 (ano chuvoso), C: 2012 (ano seco).

De acordo com Kawachi et al. (2001), a partir dos dados pontuais de chuva média histórica anual e da entropia relativa é possível obter as classes de sazonalidade para uma determinada região (Figura $3 \mathrm{AB}$ ). Assim, foram determinadas quatro classes características de chuva e sua variabilidade sazonal para semiárido brasileiro (Figura 3A). De posse dos valores pontuais de chuva e entropia, os dados foram espacializados, conforme apresentado na Figura 3B, com base nos modelos de krigagem estabelecidos anteriormente. 

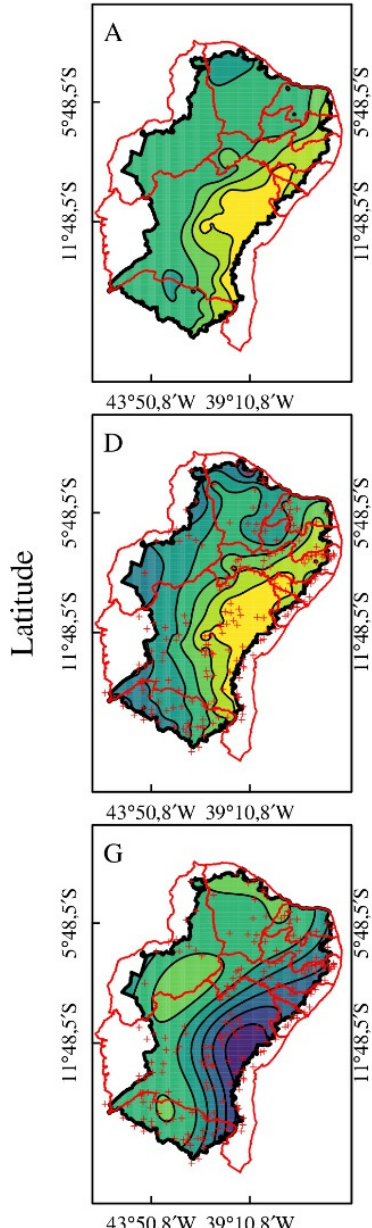

$43^{\circ} 50,8^{\prime} \mathrm{W} 39^{\circ} 10,8^{\prime} \mathrm{W}$

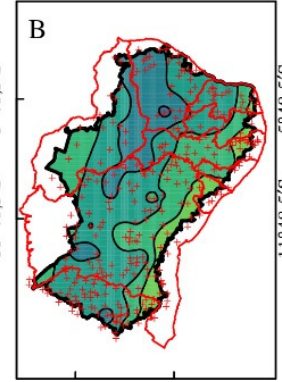

$43^{\circ} 50,8^{\prime} \mathrm{W} 39^{\circ} 10,8^{\prime} \mathrm{W}$

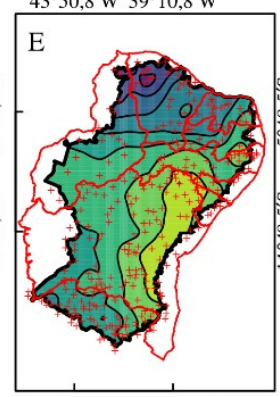

$43^{\circ} 50,8^{\prime} \mathrm{W} 39^{\circ} 10,8^{\prime} \mathrm{W}$

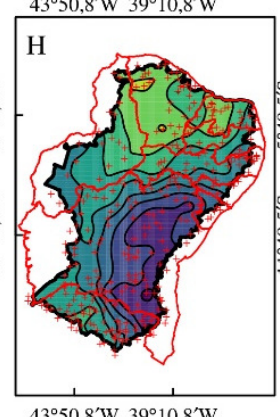

$43^{\circ} 50,8^{\prime} \mathrm{W} 39^{\circ} 10,8^{\prime} \mathrm{W}$
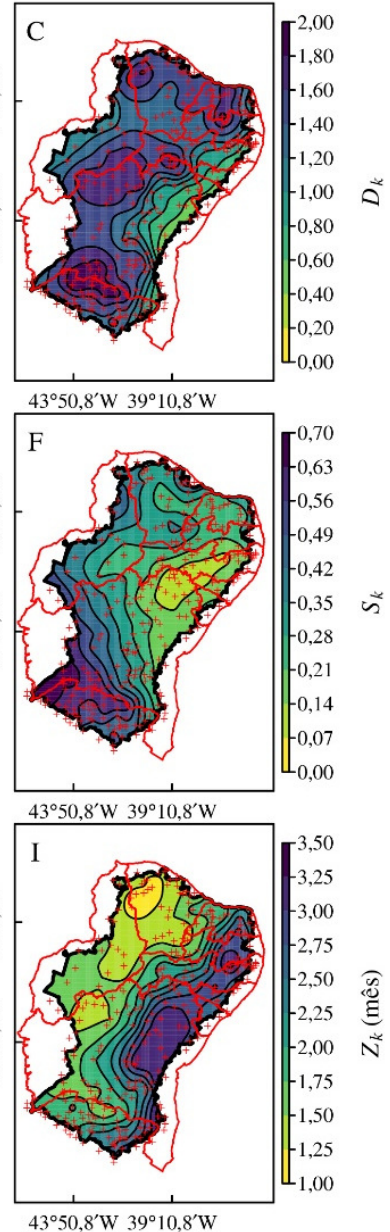

Figura 2. Distribuição espacial da entropia relativa da chuva $\left(D_{k}\right)$, índice de sazonalidade $\left(S_{k}\right)$ e duração da estação chuvosa $\left(Z_{k}\right)$ no Semiárido brasileiro para chuva média da região (A, D e G), ano chuvoso (2009 - B, E e H) e ano seco (2012 - C, F e I).
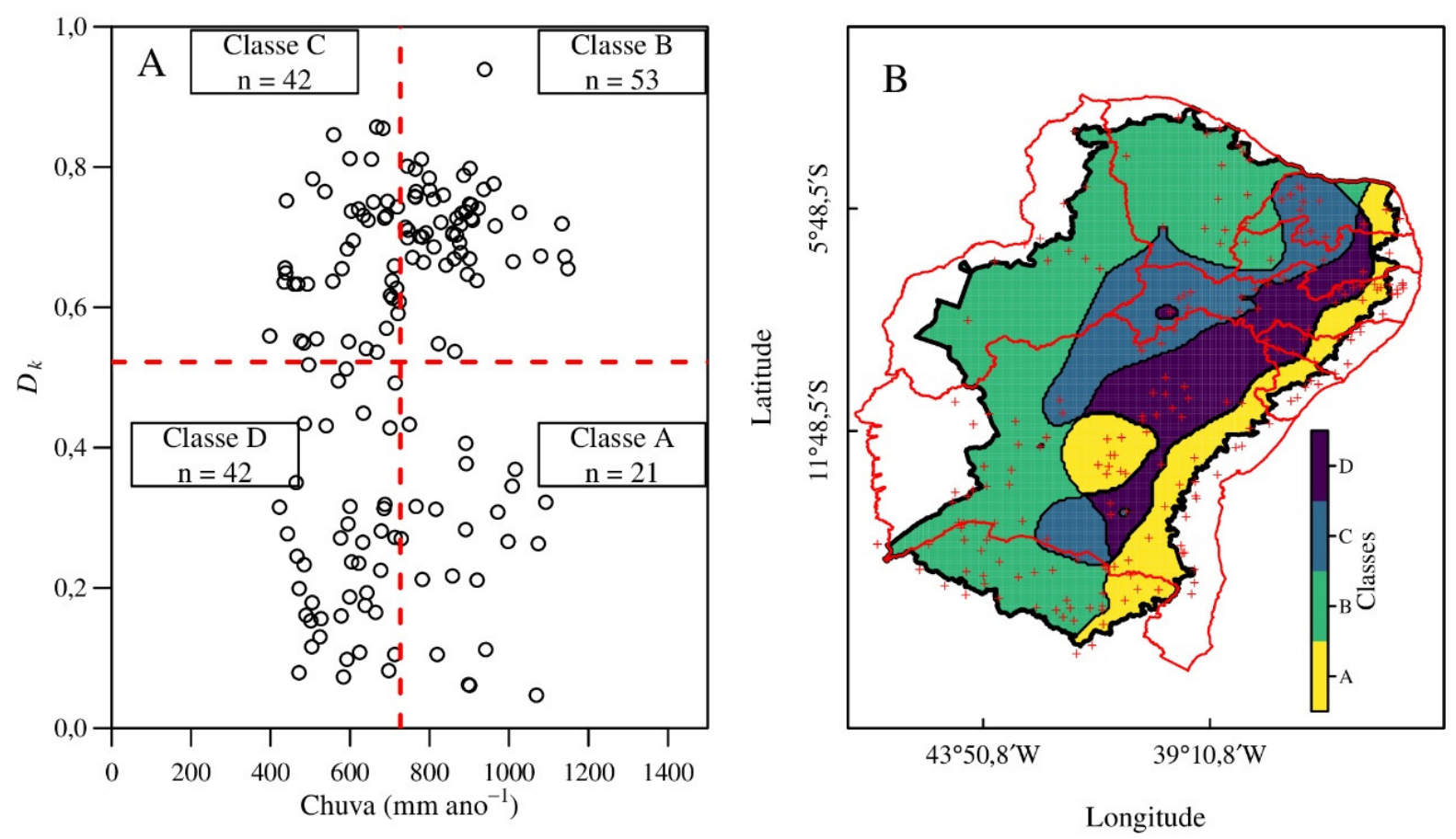

Figura 3. Classificação das condições de chuva e entropia relativa $\left(D_{k}\right)$ e distribuição espacial das classes no Semiárido brasileiro. 
As classes A, B e $\mathrm{C}$ ocorrem na mesma proporção (Figura 3B), entre 15 e $20 \%$ cada, na região semiárida, enquanto a classe $\mathrm{B}$ ocorre em cerca $47 \%$ da região (Figura 3B). A classe A, com baixa Sk e chuva acima da média da região ocorre praticamente no lado leste do semiárido brasileiro, na transição da região semiárida para agreste. $\mathrm{Na}$ outra parte da região, em direção ao oeste, ocorre quase que exclusivamente a classe B.

\section{Discussão}

A chuva média anual na região, considerando os dados históricos (normais climatológicas), foi de aproximadamente $727 \mathrm{~mm}$. Resultado semelhante foi encontrado por Nys \& Engle (2014).

Os valores estimados da entropia $\left(D_{k}\right)$ evidenciam como os eventos climáticos extremos, anos considerados secos e chuvosos, influenciam no padrão da distribuição das chuvas. Os maiores valores foram observados no ano seco, o que corrobora a má distribuição das chuvas nestes anos, com eventos de precipitação concentrados em alguns meses do ano (Mocati \& Gan, 2007). Resultado semelhante ao encontrado por Guedes et al. (2010) no estudo do uso da entropia para avaliar a disponibilidade potencial de recursos hídricos na região Nordeste do Brasil. Mesmo nos anos considerados chuvosos, com maior ocorrência de eventos de precipitação, os valores de $D_{k}$ foram maiores que os valores da média histórica, ou seja, má distribuição dos eventos de chuva ao longo do ano. O índice de sazonalidade $\left(\mathrm{S}_{\mathrm{k}}\right)$ das chuvas apresentou o mesmo comportamento do $\mathrm{D}_{\mathrm{k}}$.

Observa-se uma grande variação espacial dos dados, assim como observado por Silva (2004) e com base nos valores de entropia encontrados neste estudo. No entanto, locais com valores de chuva fora deste intervalo podem ocorrer, mas devido a escala da análise não estão representados no mapa. As áreas com menores chuvas anuais ocorrem na parte central da região semiárida, sobretudo nos Estados de Alagoas, Bahia, Pernambuco e Sergipe.

De uma forma geral, a distribuição/variabilidade espacial dos valores de chuva do ano chuvoso foi semelhante ao da normal climatológica, com os menores valores precipitados na região Norte do Estado da Bahia e região centro-oeste de $\mathrm{PE}$, assim como a parte oeste dos estados de Sergipe e Alagoas. Os locais com os maiores valores de chuva estão localizados na região limite do semiárido, principalmente no estado do Maranhão, com valores anuais acima de $1000 \mathrm{~mm}$
Em relação aos parâmetros dos semivariogramas, o alcance da chuva em 2009 foi maior do que em 2012. Uma tendência semelhante foi observada por Almeida et al. (2017), que encontraram um aumento exponencial do alcance com a chuva na escala mensal.

A entropia relativa e o índice de sazonalidade podem afetar a dinâmica do crescimento da vegetação. Segundo Souza et al. (2016), o crescimento da caatinga responde tanto ao total de chuva, como também a distribuição da chuva ao longo do ano (sazonalidade). Esses autores encontraram valores ótimos de sazonalidade para o crescimento da caatinga variando entre 0,86 e 1,32 para locais tipicamente mais chuvoso (São João-PE) e seco (Petrolina$\mathrm{PE})$, respectivamente. Os valores máximos do $S_{\mathrm{k}}$ obtidos pela krigagem tanto para o ano chuvoso, como também para o ano seco foi na ordem de 0,70 . No entanto, a interação entre o crescimento da vegetação e o valor ótimo do $S_{k}$ depende de cada local e estado da vegetação.

A entropia relativa à série de dados histórica da região foi semelhante àquela encontrada por Guedes et al. (2010) para toda a região Nordeste do Brasil. Assim como discutido anteriormente, os menores valores de entropia mostram as áreas com as menores variações dos eventos de chuva, localizadas na região centro leste da Bahia e nos Estados de Sergipe e Alagoas.

Na classe A, a chuva é acima da média da região semiárida e a entropia relativa é baixa, com uma maior duração da estação chuvosa. Na classe B, a chuva, assim como na classe A, também é acima da média e a entropia relativa também, sendo essas áreas mais propensas a eventuais cheias devido a ocorrência da estação chuvosa mais concentrada. Ou seja, regiões do semiárido onde eventos climáticos extremos (secas prolongadas e chuvas com alta intensidade) podem provocar danos significativos na agricultura. A classe $\mathrm{C}$ representa as áreas que têm índices pluviométricos abaixo da média e entropia relativa acima da média histórica do semiárido. Ou seja, uma classe/região onde precipita pouco e com alta variabilidade espacial da chuva. A classe D apresenta chuva anual abaixo da média da região e baixa entropia relativa. $\mathrm{O}$ que significa dizer que apesar do baixo valor precipitado, os eventos de chuva são bem distribuídos ao longo do tempo e do espaço.

\section{Conclusão}

As medidas estatísticas de sazonalidade e variabilidade interanual da chuva utilizadas neste estudo mostraram um desempenho satisfatório na caracterização do comportamento das chuvas na 
região semiárida do Brasil. O total anual histórico de chuva na região semiárida apresentou uma alta variação espacial e temporal, com elevados valores de entropia e baixos períodos de estação chuvosa.

A área com mais seca da região semiárida não apresentou registros de chuva acima da média no ano chuvoso, porém a chuva nessas áreas reduziu no ano seco. A duração da estação chuvosa permanece praticamente constante na parte sudeste da região semiárida independentemente do ano chuvoso ou seco. Os maiores valores de $D_{k}$ encontrados em 2009, associada com a $Z_{k}$ constante, indica que nesse ano ocorre maior volume de chuva por evento.

\section{Agradecimentos}

À Agência Nacional de Águas (ANA) pela disponibilidade dos dados de chuva. Ao Observatório Nacional da Dinâmica da Água e de Carbono no Bioma Caatinga (ONDACBC-INCT), aprovado na chamada pública MCTI/CNPq/CAPES/FAPS $\quad \mathrm{N}^{\circ} \quad 16 / 2014$ PROGRAMA INCT e financiado pela FACEPE, CAPES (Processo No 88887.136369/2017-00) e CNPq (Processo No 465764/2014-2).

\section{Referências}

CAMBARDELLA, C. A.; MOORMAN, T. B.; PARKIN, T. B.; KARLEN, D. L.; NOVAK, J. M.; TURCO, R. F.; KONOPKA, A. E. 1994. Field-Scale Variability of Soil Properties in Central Iowa Soils. Soil science society of America Journal, v. 58, n. 5, p. 1501-1511.

CAVALCANTI, I. F. A. 2012. Large scale and synoptic features associated with extreme precipitation over South America: A review and case studies for the first decade of the 21st century. Atmospheric Research, v. 118, p. 27-40.

DE LIMA MOSCATI.; M. C.; GAN, M. 2007. Rainfall variability in the rainy season of semiarid zone of Northeast Brazil (NEB) and its relation to wind regime. International Journal of Climatology, v. 27, n. 4, p. 493-512.

FENG, X.; PORPORATO, A.; RODRIGUEZITURBE, I. 2013. Changes in rainfall seasonality in the tropics. Nature Climate Change, v. 3, p. 811-815.

GUEDES, R. V.; SOUSA, S. E S.; SOUSA, F. S. 2010. Use of entropy and clustering analysis for the evaluation of water resources potential availability in the Northeastern Brazil. Ambiente e
Agua-An Interdisciplinary Journal of Applied Science, v. 5, n. 2, p. 175-187.

HASTENRATH, S. 2012. Exploring the climate problems of Brazil's Nordeste: a review. Climatic Change, v. 112, n. 2, p. 243-251.

KAWACHI, TOSHIHIKO; MARUYAMA, TAKEO; SINGH, VIJAY P. 2001. Rainfall entropy for delineation of water resources zones in Japan. Journal of Hydrology, v. 246, n. 1, p. 3644.

MARENGO, J. A. 2010. Vulnerabilidade, impactos e adaptação à mudança do clima no semi-árido do Brasil. Parcerias estratégicas, Brasília, DF, v. 13, n. 27, p. 149-176.

NYS, E. D.; ENGLE, N. 2014. Living with the semi-arid and proactive drought management in Northeast Brazil: a new perspective. Washington, DC: World Bank Group. Available from: http://www.worldbank.org/pt/country/brazil/brief/ brazil-publications-agua-brasil-series-water.

OLIVEIRA, M. B. L.; SANTOS, A. J. B.; MANZI, A. O.; ALVALÁ, R. C. DOS S.; CORREIA, M. DE F.; MOURA, M. S. B. 2006. Trocas de energia e fluxo de carbono entre a vegetação de caatinga e atmosfera no nordeste brasileiro. Revista Brasileira de Meteorologia, v. 21, p. 378-386, 2006.

RIBEIRO JÚNIOR, P. J.; DIGGLE, P. J. geoR: Analysis of geostatistical data. Disponível em: http://CRAN.Rproject.org/package=geoR. Acesso em: 16 fev. 2015.

RODRIGUEZ, R. D. G.; SINGH, V. P.; PRUSKI, F. F.; CALEGARIO, A. T. 2015. Using entropy theory to improve the definition of homogeneous regions in the semi-arid region of Brazil, Hydrological Sciences Journal, v. 7, p. 20962109.

$\mathrm{R}$ CORE TEAM. R: A Language and Environment for Statistical Computing. Vienna, Austria, 2016. Disponível em: https://www.Rproject.org/. Acesso em 28 de fevereiro de 2017.

RODY, Y. P.; DE ALMEIDA, A. Q.; RIBEIRO, A.; SEDIYAMA, G. C.; PEZZOPANE, J. E. M. 2010. Delimitação de sítios ambientais homogêneos no Estado do Espírito Santo, com base no relevo, solo e clima. Ciência Rural, v. 40, n. 12 , p. 2493-2498. 
Journal of Environmental Analysis and Progress V. 02 N. 04 (2017) 368-376

SILVA, V. P. R. 2004. On climate variability in Northeast of Brazil. Journal of Arid Environments, v. 58, n. 4, p. 575-596.

SOUZA, R.; FENG, X.; ANTONINO, A.; MONTENEGRO, S.; SOUZA, E.; PORPORATO,
A. 2016. Vegetation response to rainfall seasonality and interannual variability in tropical dry forests. Hydrological Processes, v. 30, n. 20, p. 3583-3595. 